Hope College

Hope College Digital Commons

Faculty Publications

9-2019

\title{
Allopatric Speciation in Asia Contributed to the Diversity Anomaly between Eastern Asia and Eastern North America: Evidence from Anchored Phylogenomics of Stewartia (Theaceae)
}

Jianhua Li

Hope College, li@hope.edu

Peter Del Tredici

Harvard University

Alan R. Lemmon

Florida State University

Emily Moriarty Lemmon

Florida State University

Yunpeng Zhao

Zhejiang University

Follow this and additional works at: https://digitalcommons.hope.edu/faculty_publications

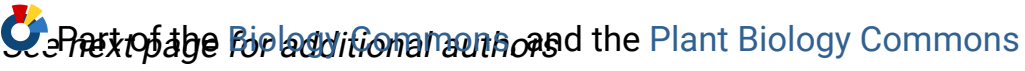

\section{Recommended Citation}

Repository citation: Li, Jianhua; Del Tredici, Peter; Lemmon, Alan R.; Moriarty Lemmon, Emily; Zhao, Yunpeng; and Fu, Chengxin, "Allopatric Speciation in Asia Contributed to the Diversity Anomaly between Eastern Asia and Eastern North America: Evidence from Anchored Phylogenomics of Stewartia (Theaceae)" (2019). Faculty Publications. Paper 1481.

https://digitalcommons.hope.edu/faculty_publications/1481

Published in: International Journal of Plant Sciences, Volume 180, Issue 7, September 1, 2019, pages 768-777. Copyright @ 2019 The University of Chicago Press, Chicago, Illinois.

This Article is brought to you for free and open access by Hope College Digital Commons. It has been accepted for inclusion in Faculty Publications by an authorized administrator of Hope College Digital Commons. For more information, please contact digitalcommons@hope.edu. 


\section{Authors}

Jianhua Li, Peter Del Tredici, Alan R. Lemmon, Emily Moriarty Lemmon, Yunpeng Zhao, and Chengxin Fu 


\title{
ALLOPATRIC SPECIATION IN ASIA CONTRIBUTED TO THE DIVERSITY ANOMALY BETWEEN EASTERN ASIA AND EASTERN NORTH AMERICA: EVIDENCE FROM ANCHORED PHYLOGENOMICS OF STEWARTIA (THEACEAE)
}

\author{
Jian-Hua Li, ${ }^{1} *$ Peter Del Tredici,† Alan R. Lemmon,‡ Emily Moriarty Lemmon,§ Yunpeng Zhao, $\|$ and Chengxin Fu\| \\ *Department of Biology, Hope College, Holland, Michigan 49423, USA; †Arnold Arboretum, Harvard University, Jamaica Plain, \\ Massachusetts 02130, USA; ¥Department of Scientific Computing, 400 Dirac Science Library, Florida State University, Tallahassee, \\ Florida 32306, USA; §Department of Biological Science, 319 Stadium Drive, Florida State University, Tallahassee, \\ Florida 32306, USA; and ॥College of Life Sciences, Zhejiang University, Hangzhou, Zhejiang 310058, China
}

Editor: Hervé Sauquet

\begin{abstract}
Premise of research. The disjunct distribution of plant genera between eastern Asia (EA) and eastern North America (ENA) has long attracted the attention of biologists and biogeographers. For most genera that have been studied, there are more species in EA than in ENA, and the diversity anomaly may have resulted from the greater physiographical heterogeneity in EA than in ENA in conjunction with climate and sea level changes. However, few empirical studies have explicitly tested the association between species diversity and allopatric speciation events. The genus Stewartia (Theaceae) displays this diversity anomaly, with two species in ENA and 21 species in EA, but the phylogeny of this group has not been resolved because of insufficient data.
\end{abstract}

Methodology. Here, we sampled 15 species of Stewartia (65\%) and generated data from over 500 nuclear loci using the anchored phylogenomic approach to produce a robust phylogeny of Stewartia. In addition, biogeographical analyses were performed to elucidate the natural history of Stewartia, including estimated times of divergence, ancestral areas, and speciation patterns.

Pivotal results. Our parsimony, Bayesian, and species tree analyses produced congruent phylogenies with high resolution of the interspecific relationships within Stewartia. Speciation in Asia was mostly allopatric between the Japanese Islands and the Asian continent during the Miocene and the early Pliocene, while the two ENA species represent lineages from different times, with $S$. malacodendron being the first lineage to split off from the remaining species and S. ovata coming later as sister to the deciduous species of Asian Stewartia.

Conclusions. The results provide direct evidence for the importance of allopatry in the differential diversity between EA and ENA.

Keywords: Stewartia, diversity anomaly, eastern Asia, eastern North America, phylogenomics.

Online enhancement: appendix. Dryad data: https://doi.org/10.5061/dryad.n4gt2qv.

\section{Introduction}

Intercontinental disjunction of plants represents one of the most important geographic patterns of biodiversity and has been studied extensively in terms of phylogenetic relationships, diversification patterns, and how and when the disjunction was formed (Thorne 1972; Raven and Axelrod 1974; Wen 1999; Milne 2006; Li et al. 2014b, 2018). Such studies have enriched theories and practices in multiple disciplines such as biogeography, ecology, and evolutionary biology (Hong 1993; Donoghue and Smith 2004; Xiang et al. 2004). Eastern Asia (EA) and eastern North America (ENA) are similar in climate and vegetation

1 Author for correspondence; email: li@hope.edu.

Manuscript received August 2018; revised manuscript received January 2019; electronically published July 26, 2019. types (Qian et al. 2017) and share numerous disjunct lineages at various taxonomic levels. At the generic level, over 65 plant genera show disjunct distribution between the two continents (Li 1952; Boufford and Spongberg 1983; Wu 1983; Wen 1999). Nevertheless, taxonomic studies of the disjunct genera have concluded that the number of species in EA is almost twice that in ENA (Li 1952; Hong 1993), a diversity anomaly between two regions with similar environmental conditions that have different species richness (Xiang et al. 2004). Various factors have been proposed to explain the diversity anomaly between EA and ENA: (1) uninterrupted connection with a tropical region in EA (Sargent 1913), (2) lack of glaciation in China proper (Hu 1935), (3) greater topographic and ecological heterogeneity (Sargent 1913; Li 1952; Axelrod et al. 1998; Qian and Ricklefs 2000; Qian 2002),

(4) longer evolutionary time (Donoghue and Smith 2004), and (5) faster rate of molecular evolution (Xiang et al. 2004). Though these factors may have acted alone or in combination to affect the 
diversification (speciation minus extinction) of lineages in EA and ENA, Qian and Ricklefs (2000) suggested that the higher number of species in EA might be associated with the greater allopatric speciation created by the isolation of populations between the Asian mainland and the Japanese Islands during the glacial and interglacial cycles and by populations adapting to different climatic zones. Phylogeographic studies of species spanning the Asian mainland and the Pacific Islands support the genetic differentiation of populations between the islands and the mainland (Qiu et al. 2009, 2011). Nevertheless, empirical evidence has rarely been used to evaluate the relative significance of the allopatric speciation and climatic adaptation hypotheses (Qian and Ricklefs 2000). In this study, therefore, we aimed at testing this hypothesis using the plant genus Stewartia L. not only because it has more species in EA than in ENA but also because it is distributed across a variety of different climatic zones in both continental Asia and the Japanese Islands.

Species of Stewartia are small to large trees (Spongberg 1974) with showy white flowers, and some are popular landscape ornamentals in the temperate regions of Eurasia and North America (Dirr 1998). Li (1996) recognized 23 species in Stewartia sensu lato (Hartia Dunn included), of which two are distributed in ENA: S. ovata (Cav.) Weatherby in the mountains of Alabama, Georgia, North Carolina, South Carolina, Tennessee, and Kentucky, and S. malacodendron L. in the piedmont of Alabama, Louisiana, Texas, Arkansas, Georgia, Florida, North Carolina, and South Carolina (fig. 1). The two ENA species differ in bracteole number and in style, leaf, and seed wing morphology (Spongberg 1974). Eight to 21 species of Stewartia have been recognized in EA, depending on (1) the number of species recognized within the $S$. sinensis Rehder \& Wilson complex in China (Spongberg 1974; Min and Bartholomew 2007) and (2) whether species of Hartia are included in Stewartia (Ye 1982; Li 1996). Three species of Stewartia are native to the Japanese Islands: S. monadelpha Siebold \& Zucc., S. psendocamellia Maxim., and S. serrata Maxim. Stewartia koreana Nakai ex Rehder is endemic to the Korean peninsula and differs from S. pseudocamellia in Japan in leaf and flower morphology and fall color

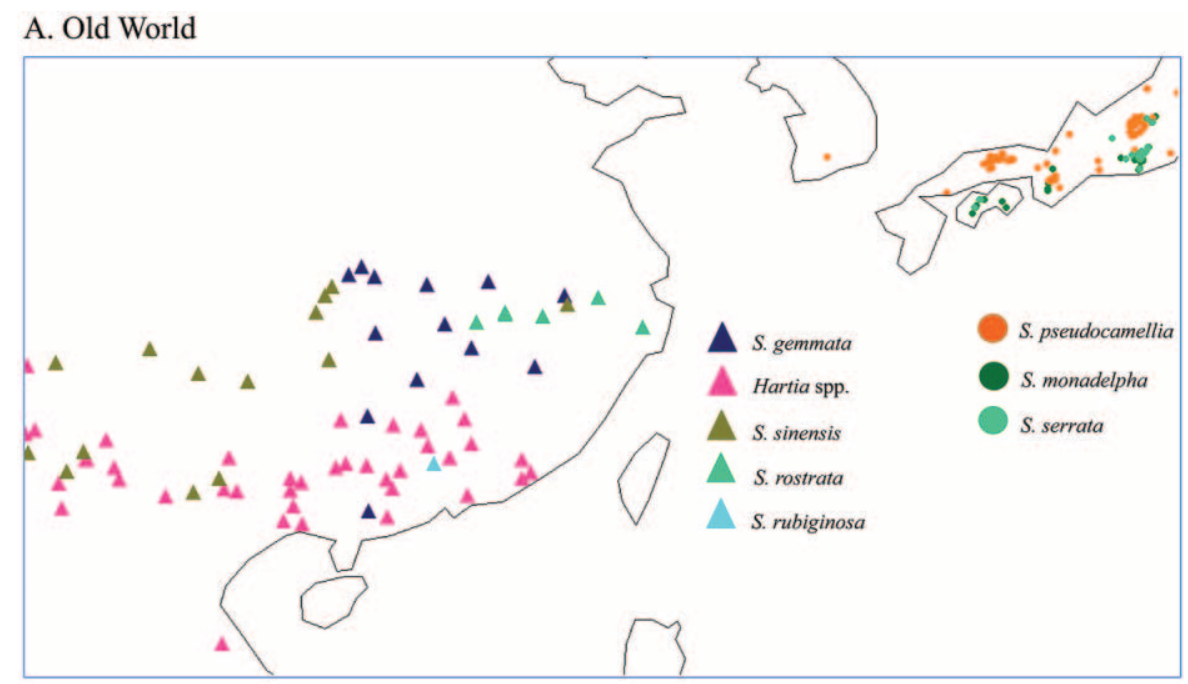

\section{B. Eastern North America}

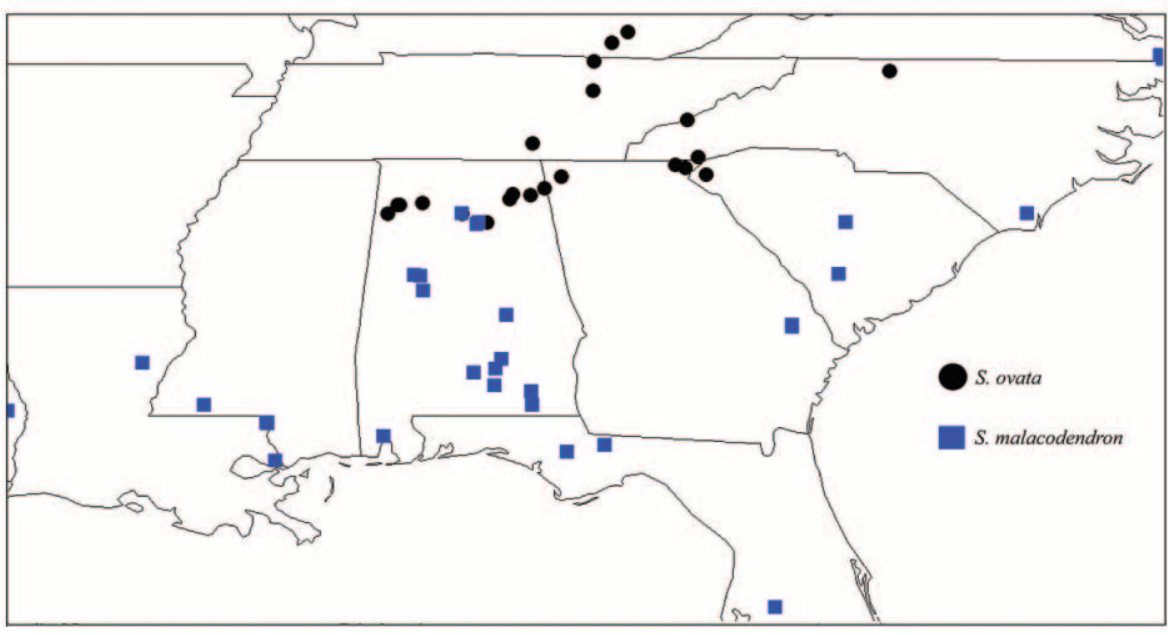

Fig. 1 Geographic distribution of species of Stewartia and Hartia based on specimen information downloaded from the Global Biodiversity Information Facility (http://www.gbif.org). 
(Rehder 1926). In China, S. rubiginosa H. T. Chang is endemic to southern Hunan and northern Guangdong, while S. rostrata Spongberg is distributed in Jiangxi, Hunan, and Zhejiang. Stewartia sinensis Rehder \& E. H. Wilson is widespread in central, northern, and southern China, and its variants have sometimes been recognized as separate species, forming a taxonomically controversial species complex (see Li et al. 2002 and references therein).

Li (1996) recognized 14 evergreen species of Stewartia in Southeast Asia. These species have naked buds (vs. deciduous species of Stewartia with bud scales) and have been recognized as a separate genus, Hartia (Ye 1982). Hartia has been divided into two morphological groups: one with solitary flowers and the other with flowers forming a racemose inflorescence (Ye 1982). Recent molecular studies support the derivation of Hartia from within Stewartia (Li et al. 2013a; Lin et al. 2019) and the taxonomic fusion of Hartia and Stewartia (Li 1996). Based on this evidence, the number of species of Stewartia in EA is over 10 times that in ENA when Hartia is included in the genus ( $\mathrm{Li}$ 1996) and at least four times that in ENA if only deciduous species are considered (Spongberg 1974; Li et al. 2002; Min and Bartholomew 2007). In this study, we treat "Hartia" as a clade of Stewartia.

If allopatric speciation has played a role in generating species of Stewartia in EA, as implied by Qian and Ricklefs (2000), we would expect a sister relationship for each island species with its respective mainland species. Similarly, if climatic disparities have selected adaptive lineages of Stewartia, it can be hypothesized that the species limited to climatic zones are closely related and share common ancestry, reflecting phylogenetic niche conservatism (Donoghue 2008). Alternatively, if sister lineages have experienced adaptations to different climatic zones, this would suggest phylogenetic overdispersion.

A well-resolved species phylogeny is essential for testing the abovementioned hypotheses, but neither nuclear internal transcribed spacers (Li et al. 2002) nor plastid genes (Li et al. 2013a) have resolved the phylogenetic relationships of the Asian species of Stewartia. Massive amounts of DNA sequence data have been shown to be informative in resolving relationships among closely related species and rapidly diversifying lineages (Pyron et al. 2014; Prum et al. 2015; Mitchell et al. 2017). The technique of anchored phylogenomics, which gathers data from hundreds of loci (Lemmon et al. 2012), has been used successfully in systematic studies of flowering plants (Buddenhagen et al. 2016; Mitchell et al. 2017). In this study, we generated large amounts of DNA sequence data via anchored phylogenomics (Lemmon et al. 2012), resolved relationships among species of Stewartia, and explored the implications of this robust phylogeny in relation to the role that allopatric speciation and climatic adaptation play in generating the diversity patterns of disjunct genera between EA and ENA.

\section{Material and Methods}

\section{Samples}

Sixty-one samples were included in this study (app. A), representing the morphological and geographic diversity of Stewartia (seven out of nine species) and "Hartia" (eight out of 14 species) and outgroups Gordonia lasianthus (L.) Ellis, Franklinia alatamaha Bartr. ex Marshall, and Schima (Li et al. 2013a). Whenever possible, multiple samples were used to account for intraspecific variation and the potential existence of incomplete lineage sorting of the nuclear loci used in the study.

\section{Molecular Techniques}

We followed Cardillio et al. (2017), Fragoso-Martinez et al. (2017), Mitchell et al. (2017), and Léveillé-Bourret et al. (2018), who targeted $\sim 500$ anchored hybrid enrichment loci using a probe kit developed by Buddenhagen et al. (2016). Briefly, genomic DNA was extracted from silica gel-dried leaves or fresh leaves using a DNeasy plant mini kit following the manufacturer's instructions (Qiagen, Valencia, CA). After quantifying DNA concentrations using Qubit fluorometric quantitation, libraries were prepared and enriched at the Florida State University (FSU) Center for Anchored Phylogenomics (Tallahassee, FL; http://www .anchoredphylogeny.com) following the methods of Lemmon et al. (2012) and Prum et al. (2015). Library preparation involved sonicating DNA to 200-800-bp fragments using a Covaris E220 focused-ultrasonicator (Woburn, MA) and ligating adapters (with 8-bp indexes) using a Beckman-Coulter Biomek FXP liquid-handling robot (Indianapolis, IN). Library pools containing approximately 16 samples each were then enriched for the anchor regions using the Agilent Angiosperm version 1 kit described in Buddenhagen et al. (2016). Enriched libraries were sequenced on two paired-end 150-bp sequencing lanes on an Illumina HiSeq2500 at the FSU Translational Science Laboratory.

\section{Data Analysis}

After sequencing, reads passing the CASAVA high-chastity filter were demultiplexed then merged following Rokyta et al. (2012). The process employed also removed sequencing adapters. The quasi-de novo assembler described by Hamilton et al. (2016) was then used to assemble reads to Arabidopsis, Billbergia, and Carex reference sequences derived from the alignments used for probed design by Buddenhagen et al. (2016). In order to avoid the effects of possible contamination and/or misindexing, assembled contigs derived from fewer than 24 reads were removed from further analysis. Pairwise distances were then used to establish the orthology of homologous contigs passing this filter, as described in Hamilton et al. (2016). After aligning sets of orthologous sequences using MAFFT (ver. 7.023b; Katoh and Standley 2013), alignments were trimmed and masked with MINGOODSITES $=14$, MINPROPSAME $=0.4$, and MISSINGALLOWED $=67$ (see Hamilton et al. 2016 for details). Misaligned regions identified upon inspection in Geneious R9 (Biomatters; Kearse et al. 2012) were masked.

Phylogenetic trees were constructed based on the concatenated data sets of all loci using the SVDquartets method (Chifman and Kubatko 2014), as implemented in PAUP* (Swofford 2002) on the CIPRES Science Gateway with 100 bootstrap replicates (Miller et al. 2010). Partitions of our data set were assessed, and their optimal models of evolution were selected using PartitionFinder (Frandsen et al. 2015). However, we did not partition our data by codon position because identifying coding regions and codon positions is difficult for this type of data (Mitchell et al. 2017). For Bayesian tree inference, two Markov chain Monte Carlo (MCMC) 
runs with four chains each were done for 10 million generations using MrBayes, as implemented on the CIPRES Science Gateway, and trees were sampled every 10,000 generations. We plotted the generations and posterior likelihood scores to ensure that the Bayesian runs had enough generations for the likelihood scores to reach the stationarity. The first $25 \%$ of generations were discarded as burn-ins. The majority consensus of the remaining trees was generated to show posterior probability support for individual clades. Species trees were inferred without concatenation of the loci using the Accurate Species Tree Algorithm (ASTRAL-II; ver. 4.9.7; Mirarab and Warnow 2015; default parameters) under the coalescent model and multilocus bootstrapping of 200 replicates from the RAxML-estimated gene trees (Feng et al. 2017). The locus-specific gene trees were estimated using RAxML version 8.1.21 (Stamatakis 2014) with GTRGAMMA model with default run settings, and branch support values were estimated using 100 bootstrap replicates.

\section{Molecular Dating}

After reviewing fossils assigned to Theaceae, Grote and Dilcher (1989) concluded that the family existed with some diversity by the Late Cretaceous. The oldest reliable fossils of Gordonia and Polyspora have been found in the Eocene of Europe and North America (Grote and Dilcher 1992). Grote and Dilcher (1992) described Gordonia lamkinensis and Gordonia warmanensis from the Middle Eocene of the Claiborne Formation in Kentucky and Tennessee, which ranges from 37.8 to $47.8 \mathrm{Ma}$ (Walker et al. 2012). We used the middle Eocene (ca. $40.4 \mathrm{Ma}$ ) as the minimal divergence age of the tribes Gordonieae and Stewartieae, as in $\mathrm{Li}$ et al. (2013a). Fruits of Schima were recently reported from the Nanlin Formation in Longchuan Basin, Yunnan, China, suggesting the minimum divergence time of Franklinia and Schima in the early to middle Miocene (ca. 17.3 Ma; Li et al. 2013b). Although fossil leaves, fruits, and seeds of "Hartia" (Mai 1975) and Stewartia (van der Burgh 1978) have been reported from the late Tertiary of Europe and Asia, none of them can be confidently identified as closely related to an extant species (Grote and Dilcher 1989). Therefore, we did not attempt to use the fossils as calibration points. Our geological timescales followed Walker et al. (2012).

Both semiparametric and parametric methods (Sanderson 2002; Drummond et al. 2016) have been widely used to estimate the time of divergence of lineages. Here, we used the r8s-PL (penalized likelihood) method (Sanderson 2002, 2003) to estimate the times of divergence of lineages within Stewartia using 100 Bayesian trees and the smoothing factor determined via cross evaluation (Sanderson 2006). The minimum age of Gordonieae and Stewartieae was set at $40.4 \mathrm{Ma}$ and that of Franklinia and Schima at 17.3 Ma. For parametric estimates of divergence times using the computer package BEAST (Drummond et al. 2016), we reduced the original data set by including a single sample for individual populations of each species and limiting the number of sites to about 50,000. The informativeness of each partition, as defined by the PartitionFinder, was evaluated and ranked in PhyDesign (Lopez-Giraldez and Townsend 2011). The top 25 partitions were used in BEAST for the dating exercises. The age priors for Gordonieae-Stewartieae and Franklinia-Schima were set at an exponential distribution with an offset of 40.4 and 17.3 Ma, respectively, and a mean of 1.0. Sites were set up with the substitution model GTRGAMMA+I with four gamma categories and estimated base frequencies. Both Yule and birthdeath speciation processes were used as tree prior models, with one of the Bayesian trees as the initial tree. Operator mix was set to fixed tree topology with tree estimates including subtreeSlide, narrowExchange, wideExchange, wilsonBalding, and subtreeLeap deselected. For the BEAST analysis, we set the length of the MCMC to 200 million generations with parameters sampled every 1000 generations to make sure that the effective sample size was over 200, as determined by Tracer (Drummond et al. 2016). The first $25 \%$ of the trees were discarded as burn-ins. The remaining trees were summarized and annotated with posterior probabilities and with highest posterior density node heights and rates using TreeAnnotator in BEAST (ver. 1.8.4; Drummond et al. 2016).

\section{Biogeographic Analysis}

Because Stewartia shows a disjunct distribution between EA and ENA and the focus of the study was on the diversification of the island lineages, we included three areas of endemism in the biogeographic analysis: continental Asia, the Japanese archipelago, and ENA. In order to infer the ancestral area of the three Japanese species, we used both statistical dispersal and vicariance analysis (S-DIVA; Yu et al. 2010) and dispersal and extinction cladogenesis (DEC; Ree and Smith 2008) analysis, as implemented in RASP (Yu et al. 2015). S-DIVA penalizes dispersal and extinction and takes into account the uncertainty of phylogenies, while the DEC method infers the likelihood of ancestral areas by incorporating models of range changes and branch lengths of the phylogeny (Ree and Smith 2008). For SDIVA, we conducted the analysis using 100 trees randomly selected from the Bayesian trees. The maximum credibility tree produced from a sample of trees in BEAST was used for the DEC analysis, wherein we assumed an equal probability of migration across the three areas of endemism in all periods of geological times because of the lack of information about the dispersal potential of seeds and fruits of Stewartia and the impact of the formation of the Sea of Japan during the early Miocene on the dispersal of Stewartia. Matzke (2014) proposed a DEC+J model to infer biogeographic history, introducing the J parameter for "founder event" or "jump dispersal" speciation, which creates a new colonization without the existence of a widespread ancestor. However, concerns about the validity of the DEC $+\mathrm{J}$ model have been raised (Ree and Sanmartín 2018), and the model was not used in this study.

\section{Results}

\section{Sequence Data and Phylogenetic Relationships within Stewartia}

Several samples were sequenced twice to ensure the accuracy of the data generated, and the sequence data were deposited in the Dryad Digital Repository (https://doi.org/10.5061/dryad .n4gt2qv; Li et al. 2019). Our sequencing targeted 500 nuclear loci and produced data from 532 orthologous loci including the flanking regions of some target loci. The concatenated data set contained 386,131 aligned nucleotide sites, with missing data ranging from $2 \%$ to $76 \%$ (mean $=20 \%$ ). Repeated sequencing of the same samples produced identical results. ParitionFinder 
recognized 206 partitions in the data set. Both SVDquartets analysis of the concatenated data set and Bayesian analyses of 206 partitions identified by the PartitionFinder produced congruent and well-supported phylogenetic trees (fig. 2). The trees are congruent with the species tree (fig. B3; figs. B1-B3 are available online) generated with ASTRAL under the coalescent model. When Gordonia, Franklinia, and Schima were used as outgroups to root the trees, Stewartia malacodendron was sister to the

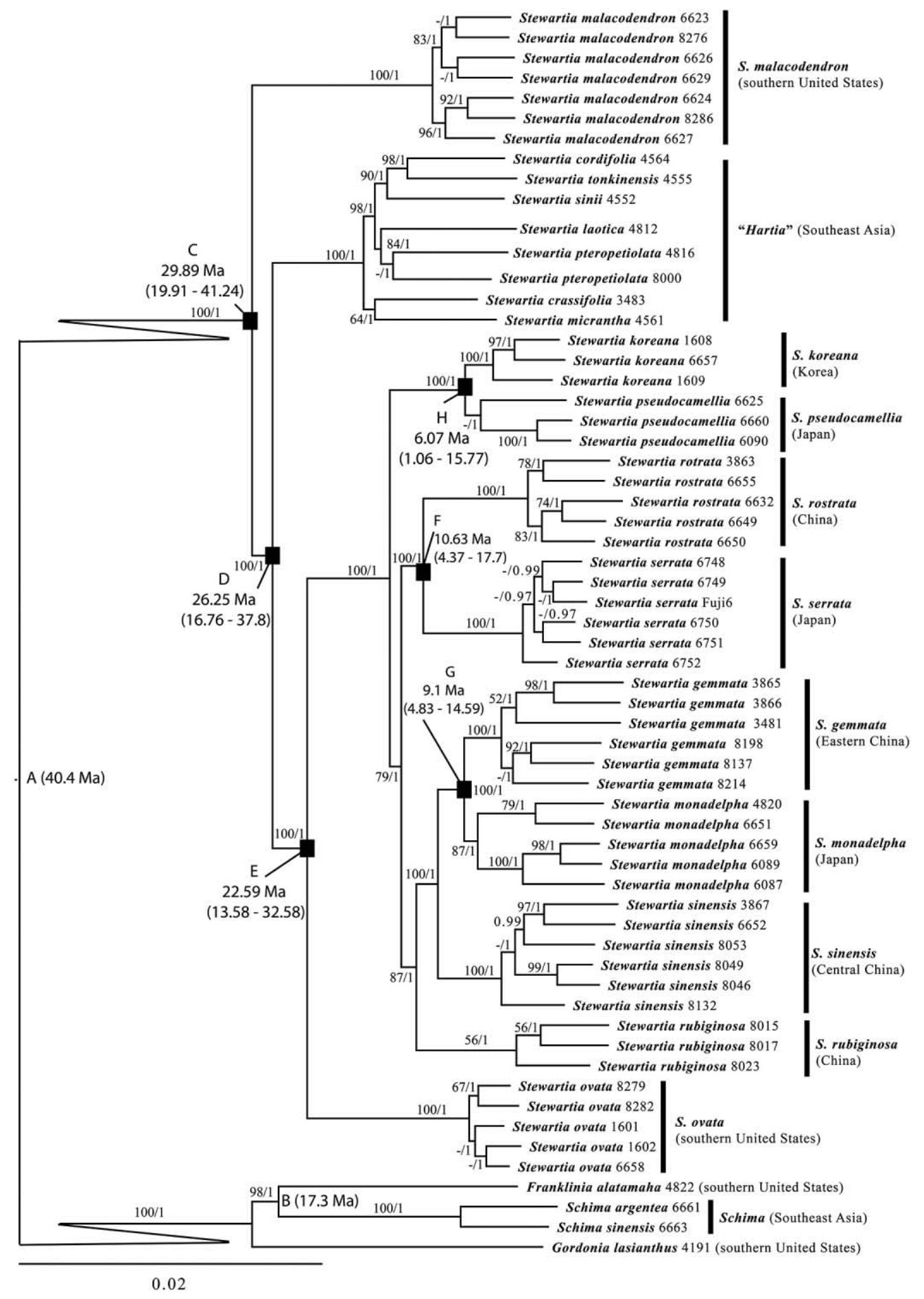

Fig. 2 Phylogeny of Stewartia generated from maximum parsimony and Bayesian analyses. Numbers at the nodes represent bootstrap percentages and posterior probabilities. Highlighted letters A and B represent nodes with fossil dates used for calibration. Letters C-H indicate nodes, with estimated times of divergence in parentheses. Japanese island species diverged from the mainland ancestral populations by the early Pliocene. 
clade containing the remaining species of Stewartia. Species of "Hartia" formed a clade and were derived from within Stewartia. Stewartia ovata was sister to the remaining deciduous Asian species of Stewartia. The deciduous Asian species of Stewartia formed a grade in the order of $S$. pseudocamellia $+S$. koreana, $S$. rostrata $+S$. serrata, $S$. rubiginosa, $S$. gemmata $+S$. sinensis, and $S$. monadelpha. Each of the three Japanese species of Stewartia formed a sister relationship with a continental species or lineage: S. pseudocamellia with S. koreana, S. serrata with $S$. rostrata, and $S$. monadelpha with $S$. gemmata.

\section{Biogeographic Patterns and Divergence Time Estimates}

Biogeographic analyses using both S-DIVA and DEC algorithms as implemented in RASP inferred that the Asian continent was the ancestral area of all three Japanese species and that three vicariance events resulted in the allopatric speciation of the Japanese lineages (fig. 3).

The cross evaluation of smoothing factors from 1 to 20 with an interval of 0.5 based on one of the Bayesian trees suggested that the optimal smoothing value for the data set was 1 . When the smoothing value and 100 Bayesian trees were used in r8s analyses, our estimated times of divergence of lineages of Stewartia ranged from 4.13 to $14.19 \mathrm{Ma}$ (table B1, available online). Specifically, the disjunction between North America and eastern Asia was formed at $11.41 \mathrm{Ma}(10.91-11.97 \mathrm{Ma}, \mathrm{SD}=0.2 \mathrm{Ma})$, and the evergreen "Hartia" evolved at $12.75 \mathrm{Ma}$ (12.22$13.25 \mathrm{Ma}, \mathrm{SD}=0.22 \mathrm{Ma})$. Stewartia serrata and $S$. rostrata diverged by the middle Pliocene at 6.95 Ma (6.47-7.47 Ma, $\mathrm{SD}=0.16 \mathrm{Ma}), S$. monadelpha and $S$. gemmata at $5.49 \mathrm{Ma}$ (5.2-5.88 Ma, SD =0.14 Ma), and S. pseudocamellia and S. koreana at $4.13 \mathrm{Ma}(3.86-4.4 \mathrm{Ma}, \mathrm{SD}=0.12 \mathrm{Ma})$.

Dating using BEAST with Yule and birth-death tree models produced age estimates with a wide range of variation for EAENA disjunction and the three species pairs between the Japanese Islands and the Asian continent (table B1). Stewartia ovata diverged from the Asian species at $22.59 \mathrm{Ma}$ (13.58-32.58 Ma; Yule) and 22.41 Ma (13.24-32.41 Ma; birth-death model), while S. rostrata and S. serrata, S. gemmata and S. monadelpha, and S. pseudocamellia and S. koreana were separated at $10.63 \mathrm{Ma}$ (4.37-17.7 Ma) and 10.46 Ma (4.26-18.09 Ma), 9.1 Ma (4.8314.59 Ma) and 9.09 $\mathrm{Ma}(4.18-15.26 \mathrm{Ma})$, and 6.07 Ma (1.0615.77 Ma) and 6.11 Ma (1.23-14.42 Ma), respectively (figs. B1, B2, table B1)

\section{Discussion}

The diversity anomaly between EA and ENA has been noted in the 1900s (Li 1952; Hong 1993; Wen 1999) and quantitatively confirmed in the 2000s (Qian and Ricklefs 2000; Qian et al. 2017). The "out of Asia" hypothesis (Donoghue and Smith 2004) suggests that the anomaly may have resulted from more ancient ages for the Asian lineages to diversify in EA than in ENA. The higher diversity in EA may also be associated with the higher genetic diversity (Xiang et al. 2004) and/or the local origin of the EA endemic species (Harris et al. 2013), partially due to the greater physical heterogeneity of the region (Qian and Ricklefs 1999). The allopatric speciation between the Pacific
Islands and the Asian continent may have contributed to the higher diversification of species in EA than in ENA (Qian and Ricklefs 2000). Robust phylogenies of the EA-ENA disjunct genera are needed for testing the allopatry hypothesis.

The ancestral populations of Stewartia were inferred to have a widespread distribution in EA and in ENA (fig. 3). Stewartia malacodendron is the first lineage of the genus, while $S$. ovata represents one of the more recent lineages and is more closely related to the Asian species of Stewartia. Thus, the two North American species evolved at different times. Also, S. ovata occurs in mountains and adjacent piedmont with higher elevation and drier conditions, while $S$. malacodendron is native to the coastal plain and piedmont of lower elevation (Spongberg and Fordham 1975). Geographically, S. ovata is distributed in slightly higher latitude $\left(35^{\circ} \mathrm{N}\right)$ than $S$. malacodendron $\left(32^{\circ} \mathrm{N}\right.$; fig. $\left.1 B\right)$. Therefore, the two North American species arose at different times with distinctive ecology and morphology.

In EA, the Japanese island species are derived from the ancestral populations in the Asian continent (fig. 3). In two of the three cases, however, the island species occur in slightly higher latitudes than the continental sister species; the average latitudes for $S$. monadelpha and S. serrata are $34.8^{\circ}$ and $35^{\circ} \mathrm{N}$, respectively, whereas those of $S$. gemmata of eastern and southern China and S. rostrata are $28.5^{\circ}$ and $29^{\circ} \mathrm{N}$, respectively. Nevertheless, the ecological conditions of the Japanese Islands and the Asian continental species of Stewartia are similar, with warm, temperate, moist conditions (Qian and Ricklefs 2000). Therefore, adaptations to different climates may not have played an important role in the diversification of species of Stewartia in EA or in ENA. Hartia occurs in warmer regions of the Asian continent (fig. 1), but it does not form a sister relationship with any of the other Asian species of Stewartia in the northern latitudes (fig. 3). Thus, climatic zoning in Asia does not seem to have had an evident impact on the diversification of lineages of Stewartia. In contrast, the geographic barriers and connections associated with the formation of the Sea of Japan and the glacial/interglacial cycles may have been more important in the generation of greater species diversity in EA than in ENA.

Geological studies have shown that the Sea of Japan was formed in the early Miocene (18-32 Ma; Tamaki et al. 1992), and the last land connections between the Japanese Islands and the Asian continent were severed in the late Pliocene because of the glacial lowering of sea levels (Haq et al. 1987; Maruyama et al. 1997). The estimated times of divergence using r8s and BEAST are different, with the former being more recent (see above), which seems to be consistent with other studies (Nie et al. 2008), as well as with simulation studies (Ho et al. 2005). Nevertheless, the estimates are generally within the range of previous age estimates and fossil dates. For example, a fossil species of Hartia was reported from the Upper Miocene of western Europe (Mai 1975), which agrees with our estimated divergence of "Hartia" at 12.75 Ma (PL) and 16.76-37.8 Ma (BEAST; figs. 2, 3). The disjunction between $S$. ovata and Asian species of Stewartia is estimated to have formed at $11.41 \mathrm{Ma}(\mathrm{PL})$ and $13.58-32.58 \mathrm{Ma}$, which is similar to Li et al.'s (2013a) estimate of 11.3-29.1 Ma. Li et al. (2013a) did not estimate times of divergence among the Asian species of Stewartia because of the poor resolution of interspecific relationships. However, our estimates from r8s and BEAST dating exercises overlap and suggest that the Japanese species diverged from the continental ancestral populations 


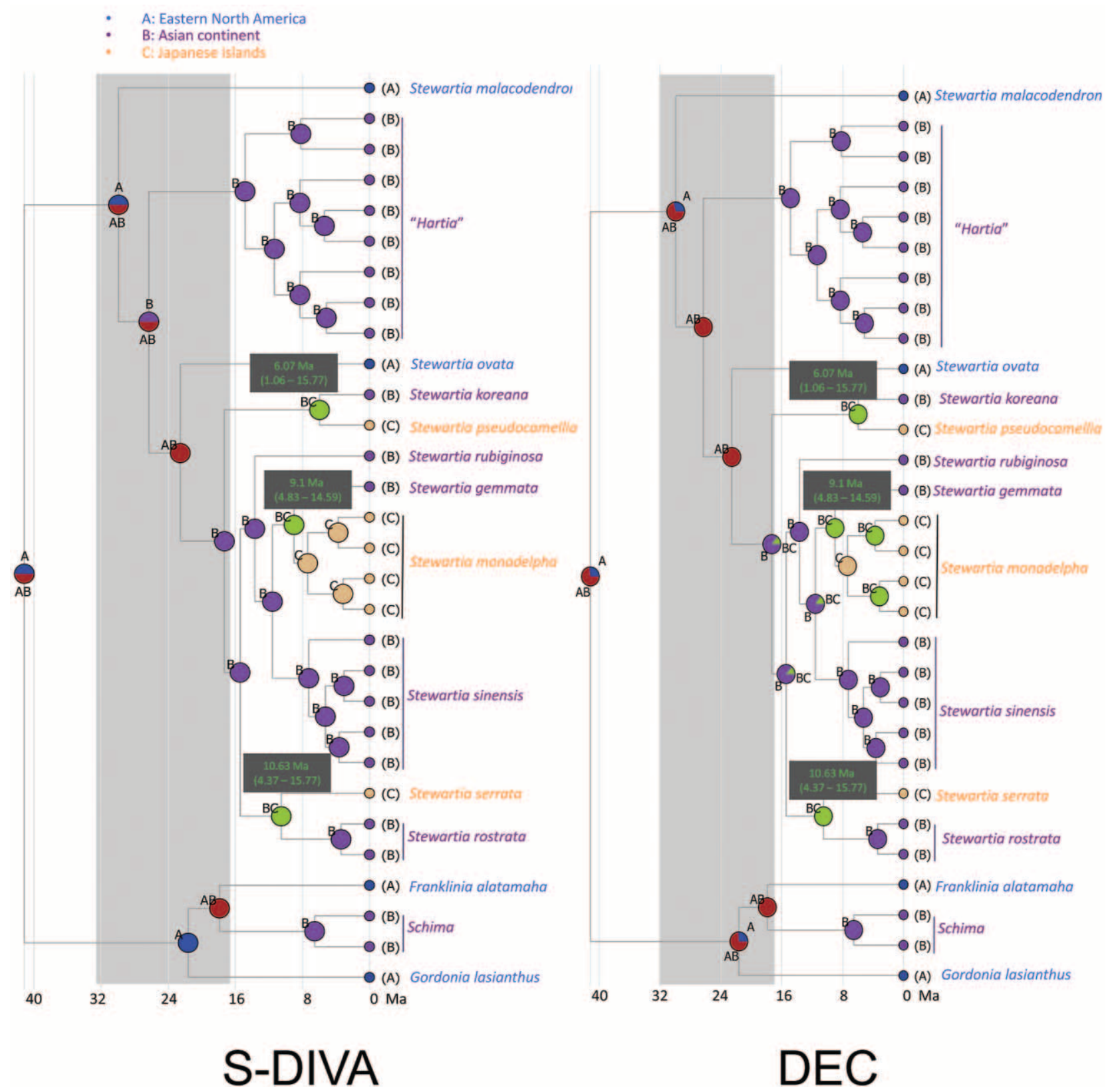

Fig. 3 Inferred ancestral areas of lineages in Stewartia from biogeographic analyses using statistical dispersal and vicariance analysis (S-DIVA) and dispersal and extinction cladogenesis (DEC) methods. Lineages on the Japanese Islands evolved from ancestral populations in the Asian continent after the formation of the sea barrier separating the islands. The three areas of endemism are eastern North America (A), Asian continent (B), and Japanese Islands (C). The vertical, wide gray bar indicates the time of formation of the Sea of Japan, 18-32 Ma.

before the late Pliocene, the most recent time when the two areas were connected via land bridges (Maruyama et al. 1997; Kameda and Kato 2011). Therefore, our phylogenetic and biogeographic analyses recognize that allopatric speciation due to the formation of the Sea of Japan was probably the most important contributing factor to the greater diversification of Stewartia in Asia, while greater climatic disparity seems to have contributed little to the diversity anomaly between EA and ENA. This is consistent with
Qian and Ricklefs (2000) and the sister relationship of the Japanese species with mainland species in several other EA-ENA disjunct lineages such as Torreya (Li et al. 2001), Tsuga (Havill et al. 2008), Pieris (Li et al. 2009), and Wisteria (Li et al. 2014a). However, many EA-ENA disjunct lineages with species on the Japanese Islands and the Asian continent need to be evaluated with better phylogenetic resolution and comprehensive taxon sampling to further test the hypothesis that there is significantly more 
allopatric speciation in EA than in ENA. It is worth noting that in the clade of $S$. gemmata, S. monadelpha, and S. sinensis, our DEC analysis suggests the possibility of $S$. monadelpha going back to the Asian continent from the Japanese Islands (fig. 3). Interestingly, a report of the introgression of plastid genes between the continental and the island species of Stewartia (Lin et al. 2019) appears to be consistent with our DEC results. Thus, a more comprehensive sampling at the population levels of all the species with molecular data from nuclear and plastid genomes will be important for gaining a better understanding of plant speciation in eastern Asia.

\section{Acknowledgments}

We thank the Arnold Arboretum, Dr. Yoko Saito, and Wenbo Liao and Qiang Fan of Zhongshan University for providing leaf material for the study; Janine Luke and Mel Seiden for providing financial support for the project; Michelle Kortyna, Jesse Cherry, Sean Holland, Alyssa Bigelow, and Kirby Birch from Florida State University's Center for Anchored Phylogenomics for their assistance with molecular data collection and analysis; and Dr. Thomas Bultman for reading the earlier version of the manuscript and providing constructive comments.

\section{Appendix A}

\section{Accession, Collection Location, and Voucher Specimen of Species and Samples Used in the Study}

AA: Arnold Arboretum; SYSU: Sunyatsen University; HCHM: Hope College.

Franklinia alatamaha Marshall, 4822, 2428-2A (AA), cultivated, USA; Gordonia lasianthus (L.) E. Ellis, 4191, North Carolina, USA, Li 4191 (AA); Stewartia cordifolia (Li) J. Li et Ming, 4564, Guangxi, China, Fan Q. 8181 (SYSU); Stewartia crassifolia (S.Z. Yan) J. Li et Ming, 3483, Guangdong, China, Li 3483 (HCHM); Stewartia laotica (Gagnep.) J. Li et Ming, 4812, Yunnan, China, Fan, Q. 8371 (SYSU); Stewartia micrantha (Chun) Sealy, 4561, Guangdong, China, Fan Q. 8150 (SYSU); Stewartia sichuanensis (S.Z. Yang) J. Li et Ming, 4816, Sichuan, China, Fan, Q. 8412 (SYSU); Stewartia pteropetiolata W.C. Cheng, 8000, Kunming, China (cultivated), Li Pan 8000 (ZJU); Stewartia sinii (Wu) Sealy, 4552, Guangxi, China, Fan Q. 8241 (SYSU); Stewartia tonkinensis (Merr.) C.Y. Wu, 4555, Guangdong, China, Fan Q. 8120 (SYSU); Schima argentea E.Pritz. ex Diels, 6661, Sichuan, China, 1992.381B (QA); Schima sinensis (Hemsl. \& E.H.Wilson) Airy Shaw, 6663, Sichuan, China, 1991.339N (QA); Stewartia gemmata S.S.Chien \& W.C.Cheng, 3865, Nanjing, Jiangsu, China, 531-34C (AA); Stewartia gemmata, 3481, Mangshan, Hunan, China, Li 3481 (HCHM); Stewartia gemmata, 8137, Kaihua, Zhejiang, China, Li 8137 (ZJU); Stewartia gemmata, 8198, Shangrao, Jiangxi, China, Li 8198 (ZJU); Stewartia gemmata, 8214, Linan, Zhejiang, China, Li 8214 (ZJU); Stewartia gemmata, 3866, Nanjing, Jiangsu, China, 531-34B (AA); Stewartia koreana Nakai, 6657, Mt. Chirisan, Korea; 11440A (AA); Stewartia koreana, 1609, Mt. Mudung, Korea; $1269-$ 83A (AA); Stewartia koreana, 1608, Mt. Chirisan, Korea; 11440B (AA); Stewartia malacodendron L., 6627, Alabama, USA; $2007-$ 149C (AA); Stewartia malacodendron, 6626, Alabama, USA; 2007-150C (AA); Stewartia malacodendron, 6624, Alabama, USA; 2007-151C (AA); Stewartia malacodendron, 6623, Alabama, USA; 2007-147B (AA); Stewartia malacodendron, 8286, Blount Co., Alabama, USA; del Tredici (AA); Stewartia malacodendron, 827, Blount Co., Alabama, USA; del Tredici (AA); Stewartia malacodendron, 6629, Virginia, USA; 2007-152C (AA); Stewartia monadelpha Siebold \& Zucc., 6087, Yakushima, Japan; 1989.319C (QA); Stewartia monadelpha, 6651, Japan, 653-74B (AA); Stewartia monadelpha, 4820, Japan, 653-74A (AA); Stewartia monadelpha, 6089, Yakushima, Japan, 1989.319D (QA); Stewartia monadelpha, 6659, Yakushima, Japan, 1989.319D (QA); Stewartia ovata (Cavanilles) Weatherby, 6658, Highlands, North Carolina, USA; 18244C (AA); Stewartia ovata, 1602, Highlands, North Carolina, USA, 18244B (AA); Stewartia ovata, 1601, USA, 18847A (AA); Stewartia ovata, 8282, Chattooga River, South Carolina, USA, (AA); Stewartia ovata, 8279, Chattooga River, South Carolina, USA, (AA); Stewartia pseudocamellia Maxim., 6625, Honshu, Japan, 2007-502A (AA); Stewartia pseudocamellia, 6090, Honshu, Japan; 1989.071A (AA); Stewartia pseudocamellia, 6660, Honshu, Japan, 1989.071A (QA); Stewartia rostrata Spongberg, 6649, Lushan, Jiangxi, China, 249-2005B (AA); Stewartia rostrata, 3863, Lushan, Jiangxi, China, 769-36A (AA); Stewartia rostrata, 6655, Lushan, Jiangxi, China, 322-2004A (AA); Stewartia rostrata, 6632, Lushan, Jiangxi, China, 249-2005A (AA); Stewartia rostrata, 6650, Lushan, Jiangxi, China, 327-2004A (AA); Stewartia rubiginosa H. T. Chang, 8017, Yingde, Guangdong, China, Li 8017 (ZJU); Stewartia rubiginosa, 8023, Ruyuan, Guangdong, China, Li 8023 (ZJU); Stewartia rubiginosa, 8015, Yingde, Guangdong, China, LI 8015 (ZJU); Stewartia serrata Maxim., 6749, Fuji Mountains, Japan; Saito 01 (TI); Stewartia serrata, 6750, Fuji Mountains, Japan, Saito 02 (TI); Stewartia serrata, 6751, Fuji Mountains, Japan; Saito 03 (TI); Stewartia serrata, 6752, Fuji Mountains, Japan, Saito 04 (TI); Stewartia serrata, fuji6, Fuji Mountains, Japan, Saito 06 (TI); Stewartia serrata, 6748, Fuji Mountains, Japan, Saito 05 (TI); Stewartia sinensis Rehder \& Wilson, 3867, Wudangshan, Hubei, China, 691-94A (AA); Stewartia sinensis, 8046, Foping, Shangxi, China, Li 8046 (HCHM); Stewartia sinensis, 8049, Foping, Shangxi, China, Li 8049 (HCHM); Stewartia sinensis, 8053, Yichang, Hubei, China, Li 8053 (ZJU); Stewartia sinensis, 8132, Badagongshan, Hunan, China, Li 8132 (ZJU); Stewartia sinensis, 6652, Shennongjia, Hubei, China, 383-2001A (AA).

\section{Literature Cited}

Axelrod DI, I Al-Shehbaz, PH Raven 1998 History of the modern flora of China. Pages 43-55 in C-I Wu, SK Wu, eds. Floristic characteristics and diversity of East Asian plants. Proceedings of the 1st International Symposium on Florisitic Characteristics and Diversity of East Asian Plants, Kunming, July 25-27, 1996. Springer, Beijing.
Boufford DE, SA Spongberg 1983 Eastern Asian-eastern North American phytogeographical relationships - a history from the time of Linnaeus to the twentieth century. Ann Mo Bot Gard 70:423-439.

Buddenhagen CE, AR Lemmon, EM Lemmon, J Bruhl, J Cappa, WL Clement, MJ Donoghue, et al 2016 Anchored phylogenomics of 
angiosperms. I. Assessing the robustness of phylogenetic estimate. BioRxiv. doi:101101/086298.

Cardillo M, P Weston, P Olde, Z Reynolds, A Mast, EM Lemmon, AR Lemmon, L Bromham 2017 The phylogeny and biogeography of Hakea (Proteaceae) reveals the role of biome shifts in a continental plant radiation. Evolution 17:1928-1943.

Chifman J, L Kubatko 2014 Quartet inference from SNP data under the coalescent model. Bioinformatics 30:3317-3324.

Dirr MA 1998 Manual of woody landscape plants. Stipes, Champaign, IL.

Donoghue MJ 2008 A phylogenetic perspective on the distribution of plant diversity. Proc Natl Acad Sci USA 105:11549-11555.

Donoghue MJ, SA Smith 2004 Patterns in the assembly of temperate forests around the Northern Hemisphere. Philos Trans R Soc B 359:1633-1644.

Drummond AJ, A Rambaut, M Suchard 2016 Beast: Bayesian evolutionary analysis by sampling trees. BEAST version 1.8.4. http://beast .bio.ed.ac.uk.

Feng YJ, DC Blackburn, D Liang, DM Hillis, DB Wake, DC Cannatell, P Zhang 2017 Phylogenomics reveals rapid, simultaneous diversification of three major clades of Gondwanan frogs at the CretaceousPaleogene boundary. Proc Natl Acad Sci USA 114:E5864-E5870.

Fragoso-Martinez I, GA Salazar, M Martinez-Gordillo, S Magallon, L Sanchez-Reyes, EM Lemmon, AR Lemmon, F Sazatornil, CG Mendoza 2017 A pilot study applying the plant anchored hybrid enrichment method to New World sages (Salvia subgenus Calosphace; Lamiaceae). Mol Phylogenet Evol 117:124-134.

Frandsen PB, B Calcott, C Mayer, R Lanfear 2015 Automatic selection of partitioning schemes for phylogenetic analyses using iterative $k$-means clustering of site rates. BMC Evol Biol 15:13. doi:10.1186 /s12862-015-0283-7.

Grote PJ, DL Dilcher 1989 Investigations of angiosperms from the Eocene of North America: a new genus of Theaceae based on fruit and seed remains. Bot Gaz 152:190-206.

1992 Fruits and seeds of tribe Gordonieae (Theaceae) from the Eocene of North America. Am J Bot 79:744-753.

Hamilton CA, AR Lemmon, EM Lemmon, JE Bond 2016 Expanding anchored hybrid enrichment to resolve both deep and shallow relationships within the spider tree of life. BMC Evol Biol 16. doi:10.1186 /s12862-016-0769-y.

Haq BU, J Hardenbol, PR Vail 1987 Chronology of fluctuating sea levels since the Triassic. Science 235:1156-1167.

Harris AJ, J Wen, J Xiang 2013 Inferring the biogeographic origins of inter-continental disjunct endemics using a Bayes-DIVA approach. J Syst Evol 51:117-133.

Havill NP, CS Campbell, TF Vining, B LePage, RJ Bayer, MJ Donoghue 2008 Phylogeny and biogeography of Tsuga (Pinaceae) inferred from nuclear ribosomal ITS and chloroplast DNA sequence data. Syst Bot 33:478-489.

Ho SYW, MJ Phillips, AJ Drummond, A Cooper 2005 Accuracy of rate estimation using relaxed-clock models with a critical focus on the early metazoan radiation. Mol Biol Evol 22:1355-1363.

Hong D-Y 1993 Eastern Asian-North American disjunctions and their biological significance. Cathaya 5:1-39.

Hu HH 1935 A comparison of ligneous flora of China and eastern North America. Bull Chin Bot Soc 1:79-97.

Kameda YC, M Kato 2011 Terrestrial invasion of pomatiopsid gastropods in the heavy-snow region of the Japanese archipelago. BMC Evol Biol 11:118. doi:10.1186/1471-2148-11-118.

Katoh K, DM Standley 2013 MAFFT multiple sequence alignment software version 7: improvements in performance and usability. Mol Biol Evol 30:772-780.

Kearse M, R Moir, A Wilson, S Stones-Havas, M Cheung, S Sturrock, S Buxton, et al 2012 Geneious Basic: an integrated and extendable desktop software platform for the organization and analysis of sequence data. Bioinformatics 28:1647-1649.
Lemmon AR, SA Emme, EM Lemmon 2012 Anchored hybrid enrichment for massively high-throughput phylogenomics. Syst Biol 61:727-744.

Léveillé-Bourret E, JR Starr, BA Ford, EM Lemmon, AR Lemmon 2018 Anchored phylogenomics in angiosperms. II. Resolving rapid generic and tribal-level radiations. Syst Biol 67:94-112.

Li H-L 1952 Floristic relationships between eastern Asia and eastern North America. Trans Am Philos Soc 42:371-429.

Li J 1996 A systematic study on the genera Stewartia and Hartia (Theaceae). Acta Phytotaxon Sin 34:48-67.

Li J-H, CC Davis, MJ Donoghue, S Kelley, P Del Tredici 2001 Phylogenetic relationships of Torreya (Taxaceae) inferred from sequences of nuclear ribosomal DNA ITS region. Harv Pap Bot 6:275-281.

Li J-H, P Del Tredici, A Lemmon, EM Lemmon, Y Zhao, C Fu 2019 Data from: Allopatric speciation in Asia contributed to the diversity anomaly between eastern Asia and eastern North America: evidence from anchored phylogenomics of Stewartia (Theaceae). Int J Plant Sci, Dryad Digital Repository, https://dx.doi.org/10.5061/dryad.n4gt2qv.

Li J-H, P Del Tredici, S-X Yang, MJ Donoghue 2002 Phylogenetic relationships and biogeography of Stewartia (Camellioideae, Theaceae) inferred from nuclear ribosomal DNA ITS sequences. Rhodora 104:117-133.

Li J-H, J Jiang, CX Fu, S Tang 2014a Molecular systematics and biogeography of Wisteria inferred from nucleotide sequences of nuclear and plastid genes. J Syst Evol 52:40-50.

Li J-H, JH Jiang, H Vander Stel, A Homkes, J Corajod, K Brown, ZD Chen 2014b Phylogenetics and biogeography of Apios (Fabaceae) inferred from sequences of nuclear and plastid genes. Int J Plant Sci 175:764-780.

Li J-H, KG Murray, P Li, K Brown 2018 Differential diversifications of South American and eastern Asian disjunct genera Bocconia and Macleaya (Papaveraceae). J Syst Evol 56:25-34.

Li MM, JH Li, P Del Tredici, J Corajod, CX Fu 2013a Phylogenetics and biogeography of Theaceae based on sequences of plastid genes. J Syst Evol 51:396-404.

Li MM, JH Li, JH Jiang, CX Fu, W Judd 2009 Phylogenetics and biogeography of Pieris (Lyonieae, Ericaceae) inferred from sequences of nuclear and chloroplast genomes. Syst Bot 34:553-560.

Li Y, N Awasthi, J Yang, CS Li $2013 b$ Fruits of Schima (Theaceae) and seeds of Toddalia (Rutaceae) from the Miocene of Yunnan Province, China. Rev Palaeobot Palynol 193:119-127.

Lin H, Y Hao, J Li, C Fu, P Soltis, D Soltis, Y Zhao 2019 Phylogenomic conflicts resulting from ancient introgression following species diversification of Stewartia s.l. (Theaceae). Mol Phylogenet Evol 135:1-11.

Lopez-Giraldez F, JP Townsend 2011 PhyDesign: an online application for profiling phylogenetic informativeness. BMC Evol Biol 11:152. doi:10.1186/1471-2148-11-152.

Mai DH 1975 Über früchte und samen von Hartia dunn (Theaceae). Wiss Z Friedrich-Schiller-Univ Jena Math-Nat R 24:463-476.

Maruyama S, Y Isozaki, G Kimura, M Terabayashi 1997 Paleogeographic maps of the Japanese islands: plate tectonic synthesis from $750 \mathrm{Ma}$ to the present. Isl Arc 6:121-142.

Matzke NJ 2014 Model selection in historical biogeography reveals that founder-event speciation is a crucial process in island clades. Syst Biol 63:951-970.

Miller MA, W Pfeiffer, T Schwartz 2010 Creating the CIPRES Science Gateway for inference of large phylogenetic trees. Pages 4552 in Proceedings of the Gateway Computing Environments Workshop (GCE), New Orleans, LA, November 14, 2010.

Milne RI 2006 Northern Hemisphere plant disjunctions: a window on tertiary land bridges and climate change? Ann Bot 98:465-472.

Min TL, B Bartholomew 2007 Theaceae Flora of China. Vol 12. Missouri Botanical Garden, St. Louis.

Mirarab S, T Warnow 2015 ASTRAL-II: coalescent-based species tree estimation with many hundreds of taxa and thousands of genes. Bioinformatics 31:i44-i52. 
Mitchell N, PO Lewis, EM Lemmon, AR Lemmon, KE Holsinger 2017 Anchored phylogenomics improves the resolution of evolutionary relationships in the rapid radiation of Protea L. Am J Bot 104:102-115.

Nie ZL, J Wen, H Azurna, YL Qiu, H Sun, Y Meng, WB Sun, EA Zimmer 2008 Phylogenetic and biogeographic complexity of Magnoliaceae in the Northern Hemisphere inferred from three nuclear data sets. Mol Phylogenet Evol 48:1027-1040.

Prum RO, JS Berv, A Dornburg, DJ Field, JP Townsend, EM Lemmon, AR Lemmon 2015 A comprehensive phylogeny of birds (Aves) using targeted next-generation DNA sequencing. Nature 526:569573.

Pyron RA, CR Hendry, VM Chou, EM Lemmon, AR Lemmon, FT Burbrink 2014 Effectiveness of phylogenomic data and coalescent species-tree methods for resolving difficult nodes in the phylogeny of advanced snakes (Serpentes: Caenophidia). Mol Phylogenet Evol $81: 221-231$.

Qian H 2002 A comparison of the taxonomic richness of temperate plants in East Asia and North America. Am J Bot 89:1818-1825.

Qian H, Y Jin, RE Ricklefs 2017 Phylogenetic diversity anomaly in angiosperms between eastern Asia and eastern North America. Proc Natl Acad Sci USA 114:11452-11457.

Qian H, RE Ricklefs 1999 A comparison of vascular plant taxonomic richness in China and the United States. Am Nat 154:160-181.

2000 Large-scale processes and the Asian bias in species diversity of temperate plants. Nature 407:180-182.

Qiu YX, CX Fu, HP Comes 2011 Plant molecular phylogeography in China and adjacent regions: tracing the genetic imprints of quaternary climate and environmental change in the world's most diverse temperate flora. Mol Phylogenet Evol 59:225-244.

Qiu YX, XS Qi, XF Jin, XY Tao, CX Fu, A Naiki, HP Comes 2009 Population genetic structure, phylogeography, and demographic history of Platycrater arguta (Hydrangeaceae) endemic to east China and south Japan, inferred from chloroplast DNA sequence variation. Taxon 58:1226-1241.

Raven PH, DI Axelrod 1974 Angiosperm biogeography and past continental movements. Ann Mo Bot Gard 61:539-673.

Ree RH, I Sanmartín 2018 Conceptual and statistical problems with the DEC $+\mathrm{J}$ model of founder-event speciation and its comparison with DEC via model selection. J Biogeogr 45:741-749.

Ree RH, SA Smith 2008 Maximum likelihood inference of geographic range evolution by dispersal, local extinction, and cladogenesis. Syst Biol 57:4-14.

Rehder A 1926 New species, varieties and combinations from the herbarium and the collections of the Arnold Arboretum. J Arnold Arbor 7:239-250.

Rokyta DR, AR Lemmon, MJ Margres, K Aronow 2012 The venomgland transcriptome of the eastern diamondback rattlesnake (Cro- talus adamanteus). BMC Genom 13:312. doi:10.1186/1471-2164-13 -312 .

Sanderson MJ 2002 Estimating absolute rates of molecular evolution and divergence times: a penalized likelihood approach. Mol Biol Evol 19:101-109.

2003 R8s: inferring absolute rates of molecular evolution and divergence times in the absence of a molecular clock. Bioinformatics 19:301-302.

$2006 \mathrm{R} 8 \mathrm{~s}$ version 1.71. http://loco.biosci.arizona.edu/r8s/.

Sargent CS 1913 Introduction. Pages xvii-xxxviii in EH Wilson, ed. A naturalist in western China with vasculum, camera, and gun. Methuen, London.

Spongberg SA 1974 A review of deciduous-leaved species of Stewartia (Theaceae). J Arnold Arbor 55:182-214.

Spongberg SA, AJ Fordham 1975 Stewartias - small trees and shrubs for all seasons. Alnodia 35:165-180.

Stamatakis A 2014 RAxML version 8: a tool for phylogenetic analysis and post-analysis of large phylogenies. Bioinformatics 30:13121313.

Swofford DL 2002 PAUP*: phylogenetic analysis using parsimony (* and other methods), version 4.0b10. Sinauer, Sunderland, MA.

Tamaki K, K Suyehiro, J Allan, JC Ingle, KA Pisciotto 1992 Tectonic synthesis and implications of Japan Sea ODP drilling. Proc Ocean Drill Program Sci Results 127/128:1333-1347.

Thorne RF 1972 Major disjunctions in the geographic ranges of seed plants. Q Rev Biol 47:365-411.

van der Burgh J 1978 The Pliocene flora of Fortuna-Gasdorf. I. Fruits and seeds of angiosperms. Rev Palaeobot Palynol 26:173-211.

Walker JD, JW Geissman, SA Bowring, LE Babcock 2012 Geologic time scale v. 4.0. Geological Society of America, Boulder, CO. doi:10.1130/2012.CTS004R3C.

Wen J 1999 Evolution of eastern Asian and eastern North American disjunct distributions in flowering plants. Annu Rev Ecol Syst 30:421-455.

Wu C-I 1983 On the significance of pacific intercontinental discontinuity. Ann Mo Bot Gard 70:577-590.

Xiang Q-Y, W-H Zhang, RE Ricklefs, H Qian, Z-D Chen, J Wen, J-H Li 2004 Regional differences in rates of plant speciation and molecular evolution: a comparison between eastern Asia and eastern North America. Evolution 58:2175-2184.

Ye C-X 1982 Systematic study of Stewartia and Hartia. Acta Sci Nat Univ Sunyatseni 21:108-116.

Yu Y, AJ Harris, C Blair, XJ He 2015 RASP (reconstruct ancestral state in phylogenies): a tool for historical biogeography. Mol Phylogenet Evol 87:46-49.

Yu Y, AJ Harris, XJ He 2010 S-DIVA (statistical dispersal-vicariance analysis): a tool for inferring biogeographic histories. Mol Phylogenet Evol 56:848-850. 